\title{
Rational rationalization and System 2
}

\author{
Commentary on Cushman's "Rationalization is Rational"
}

Wim De Neys (LaPsyDE, UMR CNRS 8240, Université de Paris)

(2020) Behavioral and Brain Sciences

\begin{abstract}
In this commentary, I highlight the relevance of Cushman's target paper for the popular dual process framework of thinking. I point to the problematic characterization of rationalization in traditional dual process models and suggest that in line with recent advances, Cushman's rational rationalization account offers a way out of the rationalization paradox.
\end{abstract}

\section{MAIN TEXT}

The dual process framework (e.g., Evans \& Stanovich, 2013; Kahneman, 2011) has long conceived human thinking as an interplay of fast and intuitive processing ("System 1" thinking) and slower, more demanding deliberate processing ("System 2" thinking). The characterization of rationalization in this popular framework is often problematic.

On one hand, rationalization is typically conceived as epiphenomenal. It is considered as a mere "making-up-excuses-after-the-facts" in which reasoners post hoc look for justifications for an (often inappropriate) intuitively cued choice. For example, one might think here of classic reasoning and heuristics-and-biases tasks in which people fail to correct biasing intuitions but are afterwards all too eager to find reasons to support their (erroneous) intuition (e.g., Evans \& Wason, 1976; Kahneman, 2011). As such, rationalization would have no functional "rational" role to play in sound reasoning.

At the same time, dual process theorists also tend to characterize rationalization as a deliberate (System 2) process. Indeed, people often spend considerable time and effort to come up with justifications and rationalize their answers (Pennycook, Fugelsang \& Koehler, 2015; Wason \& Evans, 
1975). This poses a puzzle. Why would we waste scarce resources on a pointless epiphenomenon? The fact that the human cognitive miser-who typically prefers to refrain from demanding deliberationnevertheless engages in it to rationalize its behavior suggests that rationalization must serve an important function (Evans, 2019). Unfortunately, this functional role of rationalization has received little attention in traditional dual process models.

However, recent dual process work has started to hint at a possible role in social communication and argumentation (Bago \& De Neys, 2019; De Neys, 2017; Evans, 2019). A key observation is that reasoners not only rationalize incorrect intuitions but also correct ones. One intriguing finding comes from two-response studies in which reasoners first have to answer as fast as possible with the first response that comes to mind and afterwards can take the time to deliberate and give a final answer (Bago \& De Neys, 2019; Newman, Gibb, \& Thompson, 2017). Results indicate that sound reasoners do not necessarily need to deliberate to correct an initial erroneous intuition (e.g., "10 cents" in the infamous bat-and-ball problem), their initial intuitive response is often already correct. However, without subsequent deliberation, they struggle to give an explicit justification of their (correct) intuitive answer (Bago \& De Neys, 2019). In other words, good reasoners seem to intuitively know the correct response, but don't seem to know why it is correct in the absence of further deliberation. This indicates that sound reasoners do not necessarily deliberate to correct their intuition but to rationalize it and look for an explicit justification.

As Mercier and Sperber (2017) have stressed, such a justification process in which we look for explicit reasons in support of our intuitions can be critical to efficiently sway others. Clearly, if I want to convince my peers that my solution to a problem is right, I will be more successful when giving them an explicit, verifiable argument than by simply telling them that I "felt" it was right (Bago \& De Neys, 2019).

Whereas the recent dual process findings (and the work of Mercier and Sperber, 2017) point to a possible functional role of rationalization in social persuasion, Cushman's account points to an additional contribution to our own "internal" information processing. In my opinion, such "internal" and "external" functions do not need to be mutually exclusive. However, my goal here is not to comment on the specifics of Cushman's proposal. The key point I want to highlight is that by pinpointing a rational role of rationalization, Cushman's work offers dual process theorists a possible way out of the rationalization paradox.

De Neys, W. (2020). Rational rationalization and System 2. Behavioral and Brain Sciences, 43, E34. doi:10.1017/\$0140525X19002048 
As I tried to clarify, the lack of a functional account of rationalization is problematic for dual process theories. If rationalization is not rational, it would be hard to explain why we spend our dearest resources on it and still survive as a species. Cushman builds a convincing case for the rationality of rationalization. Therefore, any dual process proponent (or critic) should take note of it. My hope is that this will instigate renewed empirical research on rationalization in the dual process field.

\section{REFERENCES}

Bago, B., \& De Neys, W. (2019). The smart System 1: Evidence for the intuitive nature of correct responding on the bat-and-ball problem. Thinking \& Reasoning, 3, 257-299.

De Neys, W. (Ed.) (2017b). Dual Process Theory 2.0. Oxon, UK: Routledge.

Evans, J. S. B., \& Wason, P. C. (1976). Rationalization in a reasoning task. British Journal of Psychology, $67,479-486$.

Evans, J. S. B. (2019). Reflections on reflection: the nature and function of Type 2 processes in dualprocess theories of reasoning. Thinking \& Reasoning. Advance online publication.

Evans, J. St. B., \& Stanovich, K. E. (2013). Dual-process theories of higher cognition advancing the debate. Perspectives on Psychological Science, 8, 223-241.

Kahneman, D. (2011). Thinking, Fast and Slow. New York, NY: Farrar, Straus and Giroux.

Mercier, H., \& Sperber, D. (2017). The enigma of reason. Harvard University Press.

Newman, I., Gibb, M., \& Thompson, V. A. (2017). Rule-based reasoning is fast and belief-based reasoning can be slow: Challenging current explanations of belief -bias and base-rate neglect. Journal of Experimental Psychology: Learning, Memory, and Cognition, 43, 1154-1170.

Pennycook, G., Fugelsang, J. A., \& Koehler, D. J. (2015). What makes us think? A three-stage dual-process model of analytic engagement. Cognitive Psychology, 80, 34-72.

Wason, P. C., \& Evans, J. S. B. (1975). Dual processes in reasoning?. Cognition, 3, 141-154.

De Neys, W. (2020). Rational rationalization and System 2. Behavioral and Brain Sciences, 43, E34. doi:10.1017/S0140525X19002048 